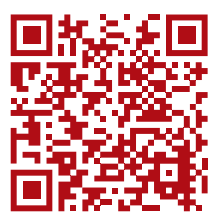

\title{
Relación de variables demográficas y presencia de labio y paladar hendido en pacientes atendidos en el Instituto Jalisciense de Cirugía Reconstructiva "Dr. José Guerrero Santos»
}

\author{
Relationship of demographic variables and presence of cleft \\ lip and palate in patients attended at the Jalisco Institute of \\ Reconstructive Surgery «Dr. José Guerrero Santos»
Dr. Omar Alejandro Ceceña-Mateos,* Dr. José Antonio Robles-Cervantes, ${ }^{\ddagger}$ Dra. Jocelyn Celeste Ledezma-Rodríguez, ${ }^{\S}$ Dr. Hiram Osiris González-Gutiérrez, Dr. Adolfo Ernesto Gómez-Díaz," Dr. Víctor Ledezma-Gómez**

Palabras clave: Labio hendido, salud pública, demografía.

Keywords: Cleft lip, public health, demographics.

\section{RESUMEN}

El labio y paladar hendido son defectos congénitos faciales que constituyen una patología importante. La etiología está asociada con múltiples factores como la genética y el ambiente. En este trabajo describimos las variables sociodemográficas de pacientes con labio y paladar hendido. Es un estudio transversal analítico por medio de una encuesta a 72 padres de pacientes sometidos a cirugía de labio y paladar hendido. La edad promedio de las madres fue de 26 años y de los padres 28 años, con promedio de edad de la madre en el primer embarazo de 19 años y un promedio de dos hijos. La posición ocupada por el hijo con labio y paladar hendido fue la segunda; $26 \%$ tenían un familiar con el mismo padecimiento; $34.7 \%$ vive en zonas rurales y $65.3 \%$ en zonas urbanas; $50 \%$ vive en zonas agrícolas. Concluimos que las condiciones previas y durante la gestación son importantes para el desarrollo del embarazo. Las gestantes tienden a ser jóvenes, con periodos intergenésicos cortos, lo que lleva a deficiencias del embarazo y por ende, a malformaciones. Éstos y otros factores son importantes para futuras investigaciones sobre el tema.

\section{ABSTRACT}

Cleft lip and palate are facial congenital defects that comprise an important pathology. The etiology is associated with multiple factors, such as: genetics and environment. In this work we describe the sociodemographic variables of patients with cleft lip and palate, in an analytical crosssectional study with a survey of 72 parents of patients undergoing cleft lip and palate surgery. The average age of the patients was 26 and of the patient's fathers was 28 , the mothers' average age in its first pregnancy of 19; they had average of two children. The position occupied by the child with cleft lip and palate was the second. $26 \%$ had a relative with the same condition. $34.7 \%$ live in rural areas; $65.3 \%$ in urban areas and $50 \%$ live in agricultural areas. We conclude that the conditions prior to and during pregnancy are important for the development of the pregnancy. Pregnant women tend to be young, and they are inclined to have short intergenesic periods, which leads to pregnancy deficiencies and therefore, malformations. These and other factors are important for future research on the subject.

ๆ Cirujano adscrito y

Director.

" Médico y Subdirector

Médico.

** Cirujano adscrito a

Cirugía Plástica.

Instituto Jalisciense de Cirugía Reconstructiva.

Recibido: 23 diciembre 2020 Aceptado: 19 abril 2021
Citar como: Ceceña-Mateos OA, Robles-Cervantes JA, Ledezma-Rodríguez JC, González-Gutiérrez HO, GómezDíaz AE, Ledezma-Gómez V. Relación de variables demográficas y presencia de labio y paladar hendido en pacientes atendidos en el Instituto Jalisciense de Cirugía Reconstructiva «Dr. José Guerrero Santos». Cir Plast. 2021; 31 (2): 56-61. https://dx.doi.org/10.35366/102746 


\section{INTRODUCCIÓN}

$\mathrm{E}^{\prime}$ labio y paladar hendido es uno de los padecimientos que ocupan los primeros lugares en las alteraciones congénitas que afectan las estructuras de la cara. Desde el punto de vista de la epidemiología, la incidencia de labio y paladar hendido (LPH) en México es de 1.1 a 1.39 por cada 1,000 nacidos vivos registrados, además de ocupar el primer lugar entre todas las anomalías. A nivel internacional, la incidencia de LPH varía en relación con la zona y raza: en la raza oriental se reporta 1.2 por cada 1,000 recién nacidos, mientras que en la negra presenta un caso por cada 2,500 nacidos. ${ }^{1-4}$

En Jalisco la incidencia de LPH es una de las más altas de todo el país; en el año 2015 la tasa de incidencia en niños de un año y menores en el Instituto Jalisciense de Cirugía Reconstructiva fue de $4.84 \%$ y el promedio anual que esta institución atiende es de un estimado de 1,200 niños con este padecimiento. ${ }^{3}$

Desde el punto de vista de la embriología, el LPH se considera una falla en la fusión de procesos faciales durante periodos cruciales en el desarrollo embrionario. ${ }^{5-7}$ La malformación se clasifica en la literatura científica por estructuras o por anatomía expuesta. ${ }^{3}$

La causa de LPH ha sido un tema de discusión y de diversas investigaciones. Se ha encontrado que es una malformación asociada a múltiples variables, de las cuales la herencia y la exposición a ciertas sustancias son las de mayor asociación, de las cuales, la de mayor porcentaje es la asociación múltiple y en menor medida sólo el antecedente familiar. 8,9

Los factores de riesgo asociados al LPH, además de los ya mencionados, son el consumo de tabaco, alcohol, estupefacientes y contaminantes en alimentos. , $^{2,10-13}$

Por otro lado, aspectos que intervienen en la salud y sus determinantes están presentes en los diferentes contextos en la vida de las personas, como el hecho de vivir en zonas remotas o en zonas urbanas y carecer de acceso a los servicios de salud. Conocer estos factores es importante para poder incidir en un cambio y prevenir, diagnosticar y tratar de mejor manera la enfermedad. ${ }^{1,14-18}$
El objetivo de este trabajo fue describir las variables sociodemográficas presentes en pacientes con labio y paladar hendido.

\section{MATERIAL Y MÉTODO}

Se realizó un estudio exploratorio analítico transversal aplicando una encuesta de elaboración propia a los padres de los pacientes con LPH que fueron intervenidos en el Instituto Jalisciense de Cirugía Reconstructiva (IJCR), la cual se integró de tres segmentos: datos personales y del embarazo, datos sociodemográficos y perfil socioeconómico. Se tuvo una muestra por conveniencia, no probabilística, ya que al ser una institución de referencia en cirugía reconstructiva, gran cantidad de personas son atendidas en este lugar. Posteriormente se realizó el análisis de los datos con el software SPSS con pruebas paramétricas así como análisis univariado con media, porcentaje, rango, mínimo y máximo.

\section{RESULTADOS}

Análisis univariado. Al evaluar la totalidad de la población de estudio en los tres segmentos se observó lo siguiente (Tabla 1):

El promedio de edad en la que los padres conciben al menor con LPH es de 26 años en las mujeres y 28 años en los hombres. El promedio de edad en la que la madre tuvo su primer embarazo fue de 19 años. El promedio de embarazos fue de dos, además de ser la misma posición del número de hijos en la que nació el paciente con LPH, es decir, de cuatro embarazos el segundo fue el que nació con LPH.

Entre los factores de riesgo durante el embarazo como el consumo de alcohol, cigarrillo, medicamentos no aptos para embarazadas o no llevar control prenatal se encontró mayor predominio de no tener esos factores de riesgo entre 94 y 96\% de las madres. En cuanto a los antecedentes de malformaciones en la familia, $31 \%$ indicó que el paciente tenía un familiar con el mismo padecimiento.

El porcentaje de personas que viven en áreas rurales es de $34.7 \%$, mientras que $65.3 \%$ vive en áreas urbanas. De igual manera, se 


\begin{tabular}{lcc}
\multicolumn{3}{c}{ Tabla 1: Características del embarazo, factores } \\
de riesgo y datos sociodemográficos.
\end{tabular}

LPH = Labio y paladar hendidos. IMSS = Instituto Mexicano del Seguro Social, SSA = Secretaría de Salud (estatal). observó que $50 \%$ de la población encuestada vive en zonas de agricultura. En el caso de vivir cerca de fábricas o industrias, $19.4 \%$ de los encuestados indicó tener alguna cerca de su domicilio. Con respecto al grado de estudios de los padres, las madres de los pacientes fueron quienes tuvieron mayor nivel educativo: $34.7 \%$ tenía bachillerato y en el caso del padre, el nivel de estudios con mayor predominio fue secundaria en $30.6 \%$ de los encuestados.

Respecto a la actividad laboral de los padres, los oficios tenían mayor predominio en 90\% (agricultor, albañil, comerciante, empleado, obrero, peón, etc.). Sólo 10\% de los encuestados tenía alguna profesión. En el caso de la actividad laboral de la madre, $69.4 \%$ indicó que se dedicaba al hogar, mientras que $30.6 \%$ tenía algún oficio (artesana, comerciante, empleada, intendente, etc.). Por otro lado, el promedio de ingreso mensual en el hogar era de 7,200 pesos. En cuanto a la afiliación a servicios de salud, $69.4 \%$ refirió tener afiliación a servicios de la secretaría de salud estatal.

Análisis geográfico. La distribución geográfica de los pacientes con LPH en el estado de Jalisco fue principalmente en las zonas urbanas de la zona metropolitana de Guadalajara, el municipio de Guadalajara tuvo el mayor porcentaje de casos. Asimismo, los municipios que tuvieron menor número de pacientes estuvieron distribuidos en el sur, norte, noroeste y centro del estado. Entre los municipios con mayor número de casos en este estudio fueron Guadalajara, Zapopan, Tonalá, Tlaquepaque y Tlajomulco, municipios que conforman la zona metropolitana de Guadalajara (Figura 1).

Análisis inferencial. Como parte del análisis estadístico bivariado o multivariado se realizó el cruce de variables de los tres segmentos. No fue un análisis por separado por cada segmento como se hizo con el análisis descriptivo, dando lugar a una interacción de todas las variables que se tenían (Tabla 2).

Resultados de las pruebas. En los resultados obtenidos en el análisis de las variables número de embarazos y posición al nacer del paciente se aplicó la prueba regresión logística; se obtuvo una significancia de $p<0.0001$, lo cual según nuestra prueba de hipótesis asumimos que existe significancia estadística, con un valor de $r$ de 0.902 en la posición al nacer, que es el segundo hijo.

Por otro lado, en el análisis de las variables de las edades de los padres al momento de la concepción del hijo que nació con LPH, se 
utilizó la prueba $t$ de Student para muestras relacionadas y encontramos un valor de $p<0.000$, por lo que aceptamos la hipótesis alterna.

Por otra parte, en el análisis bivariado de las variables se aplicó la prueba $t$ de Student para muestras relacionadas, donde se cruzaron las variables edad del primer embarazo y edad en la que se tuvo al paciente con $\mathrm{LPH}$. En la prueba de hipótesis con una significancia de $p<0.05$ se rechazó la hipótesis nula dando diferencias en los resultados.

Por último, se realizó un subanálisis de dos grupos surgidos a partir de la misma base de datos. Éstos se dividieron en zona rural y zona urbana, a partir de esta distinción se realizó un nuevo análisis de la información. Los resultados obtenidos en este análisis final no dieron ninguna significancia estadística al utilizar la prueba de regresión lineal y $\chi^{2}$ con in intervalo de confianza de $95 \%$ y una significancia de $5 \%$.

\section{DISCUSIÓN}

Parte de nuestros hallazgos coinciden con otros trabajos de investigación, como el de Padrón-García y colaboradores que muestra la proporción del sexo del paciente: el sexo masculino tiende a ser el más afectado. Por otro lado, también se coincidió con el nivel de escolaridad de los padres: la madre es quien tiene mejor nivel, que va de secundaria a licenciatura y en el caso del padre sólo tiene secundaria. Asimismo, en cuanto a las ocupaciones, las mujeres son quienes se dedican al hogar y los hombres son los que salen a trabajar y tienen ocupaciones que implican estar expuestos a diferentes contaminantes, ya que son obreros y agricultores, entre otros. ${ }^{19}$ En este mismo estudio encontramos similitudes en el tipo de casa donde habitan las familias, la mayoría vive en casa propia en zonas conurbadas.

En lo relacionado a la edad de los padres, Acosta y colaboradores revelaron que cuanto más joven sea la madre, hay una tendencia de que el primer hijo nazca con la malformación, mismo dato que encontramos en un análisis en nuestra base de datos. ${ }^{2}$ Por otro lado, Padrón-García y su equipo describen una posible hipótesis relacionada con la posición del hijo al nacer con LPH; se observó que el tercero o cuarto hijo es el que tiene mayor probabilidad. En nuestro estudio detectamos que la posición de nacimiento en los pacientes con LPH sí tiene correlación con el número de embarazos. ${ }^{19}$

Prada Flores y colaboradores llevaron a cabo un análisis de variables ambientales con las que pudieron establecer un modelo que explica en forma adecuada la presencia de LPH, que incluye la detección de pesticidas durante el primer trimestre de embarazo, con un OR de 14.3. ${ }^{20}$ Según Nivia, el medio ambiente de las zonas rurales está altamente contaminado. Las partículas procedentes del suelo, con pesticidas adheridos, inadecuadamente protegido, pueden recorrer grandes distancias Ilevando consigo contaminantes dentro de los hogares, permaneciendo por tiempo prolongado al no estar expuestas al sol, lluvia y actividad microbiana. ${ }^{20,21}$

Barrera y su equipo analizaron factores de riesgo maternos asociados al desarrollo de $\mathrm{LPH}$, donde se observó que el papel de la nutrición de la madre al momento de la gestación es importante, ya que algunos estudios reve-

Tabla 2: Análisis inferencial de variables.

\begin{tabular}{|c|c|c|c|}
\hline & \multicolumn{2}{|c|}{$r$} & $\mathrm{p}$ \\
\hline \multirow[t]{2}{*}{ Número de embarazos-posición al nacer del paciente } & \multicolumn{2}{|c|}{0.902} & 0.0001 \\
\hline & $\mathrm{t}$ & $\mathrm{gl}$ & $\mathrm{p}$ \\
\hline Edad de padres al momento de la concepción del paciente & -6.152 & 71 & 0.003 \\
\hline Edad del primer embarazo-edad embarazo paciente & -3.086 & 71 & 0.000 \\
\hline
\end{tabular}




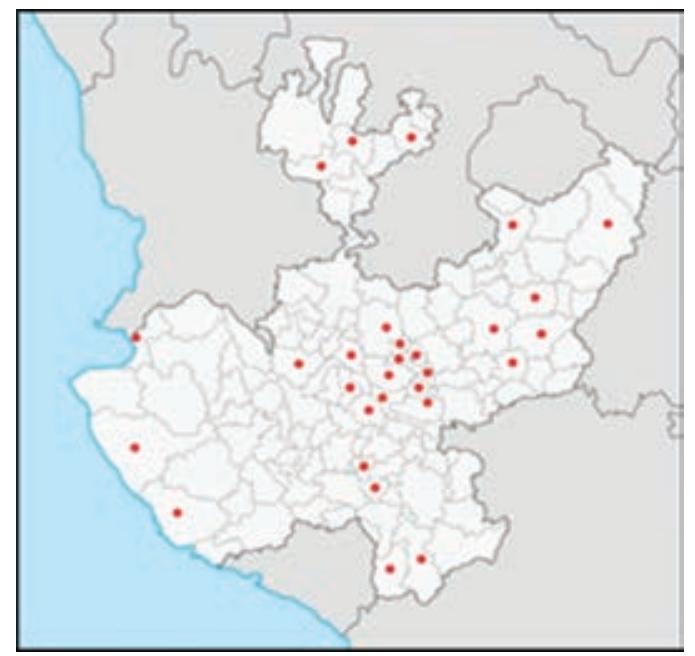

Figura 1: Distribución de casos de labio y paladar hendido en el estado de Jalisco.

Fuente: Recurso libre en línea.

laron que un nivel de zinc materno deficiente puede convertirse en un factor de riesgo sólo cuando dicho nivel está altamente comprometido. En otro estudio detectaron que un nivel bajo de zinc en la madre se asocia con el riesgo de tener un hijo con $\mathrm{LPH} .{ }^{22-24}$ Esto marca un parteaguas a futuras investigaciones, ya que desde el punto de vista gestacional las mujeres no tienen un periodo suficiente de recuperación física cuando ya están en otro periodo gestacional sin los nutrientes necesarios para satisfacer los requerimientos que conlleva este estado.

De nuestros hallazgos, es relevante mencionar la importancia de llevar a cabo investigaciones con relación a la contaminación, de residuos sólidos y pesticidas, entre otros, para un mejor entendimiento de este factor de riesgo de la malformación de labio y paladar hendido.

Como en muchos trabajos de investigación se presentan debilidades y fortalezas. Nuestras debilidades fueron que al ser un estudio transversal podemos caer en sesgos de ambigüedad temporal, es decir, que se mide la causa y el efecto al mismo tiempo. Otra limitación es no hacer distinción entre labio y paladar hendido en nuestro análisis.

Dentro de nuestras fortalezas, podemos asumir que por ser una institución de refe- rencia en cirugía reconstructiva y un hospital a población abierta, es posible incrementar el número de pacientes para continuar con este trabajo de investigación.

\section{CONCLUSIÓN}

A partir de los datos encontrados, podemos establecer ciertas consideraciones:

La malformación de labio y paladar hendido sigue siendo un padecimiento que no tiene una etiología como tal, sino que pertenece a las enfermedades con una asociación multicausal, es decir, que no es producida únicamente por un factor, sino por la presencia de dos o más factores asociados.

Así como en otros reportes, se observó que esta enfermedad sigue afectando en su gran mayoría a grupos vulnerables como los de escasos recursos, o que no tienen acceso a servicios de salud, esto se traduce en llevar un control prenatal no adecuado o nulo, de tal manera que el riesgo de desarrollar una enfermedad se incrementa.

La planificación familiar es nula de inicio o en embarazos posteriores. Las mujeres tienden a ser madres muy jóvenes, por lo que no hay una preparación adecuada del cuerpo para concebir. Por otro lado, los periodos intergenésicos son cortos, lo que lleva a no tener la recuperación adecuada física y nutricional.

La reducción de la incidencia de pacientes con malformaciones congénitas depende de una buena interacción entre diversos factores como el sistema de salud, las personas, el medio ambiente, etcétera.

\section{REFERENCIAS}

1. González-Osorio CA, Medina-Solís CE, PontigoLoyola AP, Casanova-Rosado JF, Escoffié-Ramírez $\mathrm{M}$, Corona-Tabares MG et al. Estudio ecológico en México (2003-2009) sobre labio y/o paladar hendido y factores sociodemográficos, socioeconómicos y de contaminación asociados. An Ped 2011; 74 (6): 377-387.

2. Acosta RM, Percastegi MD, Flores MB. Frecuencia y factores de riesgo en labio y paladar hendidos del Centro Médico Nacional "La Raza". Rev Mex Cir Bucal y Maxilofacial 2012; 9 (3): 109-112.

3. Pons-Bonals A, Pons-Bonals L, Hidalgo-Martínez SM, Sosa-Ferreyra CF. Estudio clínico-epidemiológico en niños con labio y paladar hendido en un hospital de 
segundo nivel. Bol Med Hosp Inf Mex 2017; 74 (2): 107-121.

4. Tirado ALR, Madera AMV, González MFD. Interacciones genéticas y epigenéticas relacionadas con fisuras de labio y paladar no sindrómicas. Av Odontoestomatol. 2016; 32 (1): 21-34.

5. Picazo JP, Gallegos MFR. Labio y paladar hendido. Conceptos actuales. Acta Med Grupo Ángeles 2019; 17 (4): 372-379.

6. Orive AAO, López ÁGB, Liceaga CCE, Chico JCE, Muñoz DAG. Labio y paladar hendido: Deleciones asociadas a la malformación congénita. Disponible en: https//unamglobal.unam.mx

7. Cruz RY, Pérez MMT, De León ONE, Suárez BF, Llanes RM. Antecedentes de enfermedades maternas en pacientes con fisura de labio y/o paladar en Ciudad de la Habana. Rev Cuba Estomatol 2009; 46: 10.

8. González CL, Bravo SP, Salgado MLL. Determinación inmunohistoquímica del factor de crecimiento epidérmico y factor de crecimiento transformante beta-3 en pacientes con labio y paladar hendido. Cir Plast 2015; 25 (1): 15-22.

9. Mena-Olalde J, González-Díaz I, Venegas-Gómez T, González-Díaz V, Medina-Aguilar S. Epidemiologia descriptiva de hendiduras labio palatinas en la Clínica de Labio y Paladar Hendidos de Morelia, Michoacán, México (1989-2012) y su comparación con algunas poblaciones internacionales. Cir Plast Iberolatinoam 2017; 43 (1): 5.

10. Ayala ACM, Vergara DES. Factores de riesgo materno predominantes asociados con labio leporino y paladar hendido en los recién nacidos. Arch Inv Mat Inf 2012; 4 (2): 55-62.

11. Nazer HJ, Ramírez RMC, Cifuentes OL. 38 Años de vigilancia epidemiológica de labio leporino y paladar hendido en la maternidad del Hospital Clínico de la Universidad de Chile. Rev Médica Chile 2010; 138(5):567-572.

12. Muñoz J, Bustos I, Quintero C, Giraldo A. Factores de riesgo para algunas anomalías congénitas en población colombiana. Rev Salud Pública 2001; 3 (3): 268-282.

13. Rojas EG, Campos JFA, Mariscal HA. Panorama epidemiológico de labio y paladar hendido en México. Cir Plast 2017; 27 (1): 10-15.

14. Pawluk MS, Campaña H, Gili JA, Comas B, Giménez LG, Villalba MI et al. Determinantes sociales adversos y riesgo para anomalías congénitas seleccionadas. Arch Argent Ped 2014; 112 (3): 215-223.

15. Beluci ML, da Silva DMCC, dos Santos TA, Spadoti DRA. Correlation between quality of life and burden of family caregivers of infants with cleft lip and palate. Rev ESC Enferm USP [Internet]. 2019 [cited 10 February 2020];53. Available in: http://www. scielo.br/scielo.php?script $=$ sci_abstract $\&$ pid $=$ S0080$62342019000100422 \& \operatorname{lng}=\mathrm{e}^{-} \& \mathrm{nrm}=$ iso\&tlng $=\mathrm{en}$
16. Chavarriaga-Rosero J, González-Caicedo MX, RochaBuelvas A, Posada-López A, Agudelo-Suárez AA. Factores relacionados con la prevalencia de labio y paladar hendido en la población atendida en el Hospital Infantil "Los Ángeles". Municipio de Pasto (Colombia). Odontología 2011; 24 (2): 33-41.

17. Acuña-González G, Medina-Solís CE, Maupomé G, Escoffie-Ramírez M, Hernández-Romano J, MárquezCorona $\mathrm{M}$ de $\mathrm{L}$ et al. Family history and socioeconomic risk factors for non-syndromic cleft lip and palate: A matched case-control study in a less developed country. Biomédica 2011; 31 (3): 381-391.

18. Pawluk MS, Campaña H, Rittler M, Poletta FA, Cosentino VR, Gili JA et al. Individual deprivation, regional deprivation, and risk for oral clefts in Argentina. Rev Panam Salud Pública 2018; 41: e110.

19. Padrón-García AL, Achirica-Uvalle M, Collado-Ortiz MA. Caracterización de una población pediátrica con labio y paladar hendidos. Cir Cir 2006; 74 (3): 159-166.

20. Prada FAM, Eljach SEM, Caballero BV, Torres MEA. Factores ambientales asociados con labio o paladar hendido no sindrómico en una población del Magdalena medio colombiano. UstaSalud 2014; 13 (1): 18-25.

21. Nivia E. Mujeres y plaguicidas: una mirada a la situación actual, tendencias y riesgos de los plaguicidas: estudio de caso en Palmira, Colombia. Cali, Colombia: Rapalmira 2000. p. 114.

22. Barrera C, Mezarobba N. Factores de riesgo materno asociados con el labio fisurado con o sin paladar hendido: una revisión. Int J Odontostomatol 2016; 10 (2): 359-368.

23. Munger RG, Tamura T, Johnston KE, Feldkamp M, Pfister R, Botto $L$ et al. Plasma zinc concentrations of mothers and the risk of oral clefts in their children in Utah. Birth Defects Res A Clin Mol Teratol 2009; 85 (2): 151-155.

24. Krapels IPC, Rooij IALM van, Wevers RA, Zielhuis GA, Spauwen PHM, Brussel W et al. Myo-inositol, glucose and zinc status as risk factors for non-syndromic cleft lip with or without cleft palate in offspring: a casecontrol study. BJOG Int J Obstet Gynaecol 2004; 111 (7): 661-668

Conflicto de intereses: Los autores declaran no tener conflicto de intereses.

Correspondencia:

Dr. Omar Alejandro Ceceña-Mateos

Calzada del Federalismo Norte Núm. 2022,

La Guadalupana, 44220,

Guadalajara, Jalisco, México.

E-mail: Isp.alejandrocecena@hotmail.com 